\title{
Evaluation of Compost and Compost tea as Promising Method for Meloidogyne Incognita Management
}

\author{
N. A. Abdel-Bary ; H. H. Hendy ${ }^{* *}$; A. H. Ashoub ${ }^{* *}$; M. Y. Yassin* and \\ G. M. Abdel-Razek ${ }^{* *}$ \\ ${ }^{*}$ Faculty of Agriculture, Cairo University \\ ${ }^{* *}$ Desert Research Centre, Cairo, Egypt
}

\begin{abstract}
Two types of compost, (plant and animal) as well as furan $10 \% \mathrm{G}$ were effective in reducing Meloidogyne incognita number of galls, nematode reproduction and fecundity. Compost was investigated as amendment for suppressing populations of Meloidogyne incognita and increasing plant vigor. The greenhouse and laboratory studies were conducted with mature compost produced in SEKEM organic farm. Compost extract was prepared by steeping $100 \mathrm{~g}$ compost in $200 \mathrm{ml}$ tap water and removing biomass with cheesecloth filtration followed by centrifugation. The supernatant was diluted I: 4 in water, sterile filtered and used as $100 \%$ compost extract treatment. In micro well as says, $M$. incognita $\mathrm{J}_{2}$ activity were inhibited by all tested compost extract concentrations (25\%, 50\%, $75 \%$ and $100 \%$ extract), with $>55 \%$ inhibition in $100 \%$ compost extract after $72 \mathrm{~h}$. (Animal and plant) Compost treatments mixed with soil at doses $25,50,100 \mathrm{~g} / \mathrm{kg}$ were greater treatment gave the best result in reducing the nematode reproduction compared with untreated check. (Animal and plant) compost suppressing final nematode population values that average $92.18 \%$ and 92.54 respectively, at doses $100 \mathrm{~g} / \mathrm{kg}$ soil compared with nematicide Furan $10 \%$ at dose $2 \mathrm{~g} / \mathrm{kg}$ suppressing final nematode population values that average $79.10 \%$. Also the used (animal and plant) compost tea at two times 2 days before inoculation and 10 days after inoculation showed the same results. The highest percentage of reduction in nematode final population $(80.59 \%)$ and $(82.90 \%)$ was noticed in concentration dose100 g/200 cm water / $\mathrm{kg}$ by animal and plant compost tea 2 days before inoculation.

All the tested materials significantly suppressed nematode final population and rate of buildup. In general there were positive correlation between the concentration used and the obtained degree of nematode management.
\end{abstract}

Key words: organic, extract, compost, compost tea, plant parasitic nematode, Meloidogyne incognita, soil amendments.

\section{Introduction}

Root-knot nematodes (Meloidogyne spp.) are among the most economically damaging genera of plant parasitic nematodes on horticultural and field crops, causing an estimated US $\$ 100$ billion loss annually (Oka et al., 2000). Meloidogyne 
incognita (Kofoid\& White) Chitwood is one of the most harmful root -knot nematode species, which infects a wide range of vegetable crops in Egypt (Ibrahim et al., 2000). Root knot nematodes are among the most difficult crop-pest to be controlled. Although chemical nematicides are effective, easy to apply and show rapid effects, they have begun withdrawn from the market owing to concerns about public health and environmental safety (Rich et al., 2004). Also, nematicides have higher cost of application, limited availability in many developing countries or their diminished effectiveness following repeated applications (Schneider et al., 2003; Ahmad and Siddiqui, 2009).

Attention on environmental safety has led to the search of alternative strategies including the use of compost, one promising management strategy that can use to suppress nematode populations and increase soil health and plant vigor composts produced from various organic residues have been reported to suppress populations of nematodes and other plant disease-causing organisms.

Recently, several studies concerned the effectiveness of compost used as soil amendments in managing root -knot nematode and compost tea extract Considerable progress in the use of compost as soil amendment for the control of plant parasitic nematodes under field conditions has been reported by (McSolery and Galher, 1995,1996, 1997; Nico, 2004; zhu et al., 2006 and Zhang, 2009).

Many studies emphasized that nematode populations were greatly suppressed after compost application (Farahat et al., 2010; Renco et al., 2010; Addabbo et al., 2011 and Sasanelli et al., 2011). Meyer et al., (2011) and Ravindra et al., (2014) they reported that compost was investigated as an amendment for suppressing populations of Meloidogyne incognita and increasing plant vigor.

\section{Material and Methods}

\section{Preparation of compost extracts}

Laboratory studies were conducted with two source of organic compost (animal and plant). Compost extract was prepared by soaking for $100 \mathrm{~g}$ compost in $200 \mathrm{ml}$ tap water and removing biomass with cheesecloth filtration followed by centrifugation $3000 \mathrm{rpm}$ for $10 \mathrm{~min}$. The extract was served as standard solution. The extract was diluted to make four concentrations (i.e. $25,50,75,100 \mathrm{mg} / \mathrm{ml}$ )

\section{Nematicidal activity}

One $\mathrm{ml}$ of nematode suspension containing 100 freshly hatched juveniles of Meloidogyne incognita was added to constant volume of extracts into petri dishes $(80 \mathrm{~mm})$ and 100 freshly hatched second stage larvae of Meloidogyne incognita in 5 $\mathrm{ml}$ distilled water as control. All dishes were incubated in an incubator at $\left(25 \pm 2 \mathrm{C}^{\circ}\right)$. After 24,48 and $72 \mathrm{~h}$ the juveniles were counted for mortality and non mortality 
under stereoscope microscope. The death of nematodes was confirmed by keeping them in tap water for $24 \mathrm{~h}$. The percent mortality was worked out from an average of three replicate.

Table (1): Plant compost analysis.

\begin{tabular}{lcc}
\hline \multicolumn{1}{c}{ Analysis } & Unit & Plant compost analysis \\
\hline weight per cubic meter & $\mathrm{Kg}$ & 660 \\
Moisture & $\%$ & 32 \\
$\mathrm{PH}(1: 10)$ & & 7.61 \\
$\mathrm{EC}(1: 10)$ & $\mathrm{Ds} / \mathrm{m}$ & 2.26 \\
Total(N) & $\%$ & .98 \\
Nitrogen alamonyumi & $\mathrm{PPm}$ & 27 \\
Nitrogen nitrate & $\mathrm{PPm}$ & -- \\
Organic matter & $\%$ & 32.0 \\
Organic Carbon & $\%$ & 18.56 \\
Ash & $\%$ & 68.0 \\
C:N & $\%$ & $1: 18.93$ \\
Total phosphorus & $\%$ & .27 \\
Total Potassium & -- & .52 \\
Weed seeds & Juveniles/200g & -- \\
Nematode & & -- \\
\hline
\end{tabular}

Table (2): Animal Compost analysis.

\begin{tabular}{lcc}
\hline \multicolumn{1}{c}{ Analysis } & Unit & Animal compost analysis \\
\hline weight per cubic meter & $\mathrm{Kg}$ & 699 \\
Moisture & $\%$ & 32 \\
$\mathrm{PH}(1: 10)$ & & 7.33 \\
EC $(1: 10)$ & $\mathrm{Ds} / \mathrm{m}$ & 2.49 \\
Total $(\mathrm{N})$ & $\%$ & 0.79 \\
Nitrogen alamonyumi & $\mathrm{PPm}$ & 43 \\
Nitrogen nitrate & $\mathrm{PPm}$ & 109 \\
Organic matter & $\%$ & 22.9 \\
Organic Carbon & $\%$ & 13.28 \\
Ash & $\%$ & 77.10 \\
C:N & & $1: 16.81$ \\
Total phosphorus & $\%$ & 0.38 \\
Total Potassium & $\%$ & 0.48 \\
Weed seeds & -- & -- \\
Nematode & Juveniles/200g & -- \\
\hline
\end{tabular}




\section{A. Compost experiment}

One month old eggplant seedlings, Solanummelongena cv. Black Balady, with uniform size were transplanted singly in $15 \mathrm{~cm}$ clay pots filled with organic soil from SEKEM farm. Each treatment was replicated three times two source of organic compost (animal and plant) were applied at rates 25,50 and $100 \mathrm{~g} / \mathrm{kg}$ soil. One week later each pot was inoculated with 2000 freshly hatched juveniles Meloidogyne incognita. Each treatment was replicated three times. All treatments were arranged in a complete randomized design under greenhouse condition at $32 \pm 5^{\circ} \mathrm{C}$. Also two control treatments were done (nematicides and untreated infected plants). Pots were watered periodically every two days. The plants were harvested after 45 days from inoculation time

\section{B. Compost tea experiment}

One month old eggplant seedlings, Solanum melongena cv. Black Balady, with uniform size were transplanted singly in $15 \mathrm{~cm}$ clay pots filled with organic soil from SEKEM farm. Pots were divided into two groups; first group received animal tea compost and second received plant tea compost. The two kinds of compost were added at rates 25, 50 and $100 \mathrm{~g} / 200 \mathrm{~cm} 3$ water for each pot as drench application two days before inoculation and ten days after inoculation. Each pot was inoculated with 2000 freshly hatched juveniles Meloidogyne incognita. Each treatment was replicated three times. All treatments were arranged in a complete randomized design on clean benches in greenhouse. Also two control treatments were done (nematicide (furan $10 \% \mathrm{G}$ at rate $2 \mathrm{gm} /$ plant) and untreated infected plants). Pots were watered periodically every two days. For each experiment, the plants were uprooted after 45 days from inoculation time.

\section{Nematode enumeration and plant growth parameters determination}

Soil of each pot was processed for nematode extraction by sieving and Baerman -pan technique (Southey, 1970). A count of second stage juveniles (J2) in soil of each pot was determined by means of Hawksley counting slide under stereoscopic microscope. Also, average numbers of eggs/egg-masses were determined by rinsing four randomizely selected egg-masses per root system of each replicate in $1 \%$ sodium hypochlorite to release eggs from egg matrix. Then, the released eggs were suspended in water and counted under stereoscopic microscope. Collected juveniles were counted. Galls and egg-masses and their indices were rated. The reduction percentage in galls formation, egg-masses production, also female's numbers were counted and juveniles' number were calculated

According to the following formula:

$$
\mathrm{R} \%=(\text { Control-Infected }) / \text { Control }) \times 100
$$


The final population and nematode build-up were calculated for all treatments.

The rate of nematode increase (R.B) was detected by dividing the nematode Final Population by the nematode Initial Population (FP/PI).

Plant growth response based on leaves number, fresh shoot and root length also dry and fresh shoot and root weights were determined and calculated for all treatments. Statistical analysis was carried out according to the procedure "Anova" recorded by Senedecor and Cochran (1980). Means of treatments were compared by Dancann's Multiple Range Test at $5 \%$ level of probability. These steps were accomplished using SPSS Program version 16.

\section{Results}

Impact of compost (animal and plant) extract at four concentration 25\%, 50\%, $75 \%$ and $100 \%$ on mortality percentage of newly hatched juveniles $\left(\mathrm{J}_{2}\right)$ of Meloidogyne incognita are depicted in table (3). As it is clear appositive correlation has observed between Meloidogyne incognita $\mathrm{J}_{2}$ mortality percentages and compost extract concentration at the three different exposure times. Meanwhile, larval mortality percentages increased with the increase of compost extract concentration from $25 \%$ up to $100 \%$.It is also evident that the tested concentrations of plant compost extract were superior over that of animals.

Compost extract in larval mortality percentages at all the time of exposure, since their values were amounted to28.57, 42.42, 49.00 and 58.82 for the plant compost extract at $25 \%, 50 \%, 75 \%$ and $100 \%$ concentrations, respectively, whereas those of animal compost extract at the same time were 16.45, 32, 35, 46 and 55.44 accompanied with 25, 50,75, 100 concentrations, respectively.

Table (3): Nematicidal effect of compost extracts on mortality percentage of Meloidogyne incognita juveniles $\left(\mathrm{J}_{2}\right)$ under different concentration at different exposure times.

\begin{tabular}{|c|c|c|c|c|}
\hline \multirow{2}{*}{ Treatment } & \multirow[b]{2}{*}{ Conc. } & \multicolumn{3}{|c|}{$\%$ Mortality juveniles $\left(\mathrm{J}_{2}\right)$} \\
\hline & & $24 \mathrm{~h}$. & 48h. & $72 \mathrm{~h}$. \\
\hline \multirow{4}{*}{ Animal compost } & 25 & 10.14 & 14.63 & 16.45 \\
\hline & 50 & 20.38 & 25.86 & 32.35 \\
\hline & 75 & 23.52 & 32.39 & 46.00 \\
\hline & 100 & 27.94 & 36.36 & 55.44 \\
\hline \multirow{4}{*}{ Plant compost } & 25 & 14.08 & 19.29 & 28.57 \\
\hline & 50 & 20.00 & 27.47 & 42.42 \\
\hline & 75 & 41.17 & 47.94 & 49.00 \\
\hline & 100 & 44.93 & 49.97 & 58.82 \\
\hline Check & 00 & 00 & 00 & 00 \\
\hline
\end{tabular}




\section{Compost treatment}

Data presented in table (4) reveals that the tested compost (animal and plant) applied at all doses significantly impaired gall formation, juveniles, eggmasses, final population as well as nematode build up and egg production comparing with untreated check. Differences in nematode reduction were obvious among and/or doses treatment, while, no significant difference between the compost type, the higher the dose the higher reduction in nematode number, and gave the best results. Animal compost suppressing final nematode population values that average $73.57 \%, 85.14 \%$ and 91.70 , at doses 25,50 and $100 \mathrm{~g} / \mathrm{kg}$ soil respectively. On the other hand, plant compost suppressing final nematode population values that average $71.92 \%, 87.98 \%$ and $92.54 \%$ at doses 25,50 and $100 \mathrm{~g} / \mathrm{kg}$ soil respectively. Furan at dose $2 \mathrm{~g} /$ pot suppressing final nematode population values that average $79.22 \%$ (fig., 1 ).

Improvement in plant growth parameters in terms of shoot and root length and weights was variable and proportioned with the compost (animal and plant) treatments at different doses (table 3), while the dose $100 \mathrm{~g} / \mathrm{kg}$ gave the best result in increment the leave number and the shoot length in all treatments of compost (animal and plant) on other hand, fresh and dry shoot weight were increased also with the dose $100 \mathrm{~g} /$ pot in all treatment, also length and fresh weights of roots were increased. All treatments recorded an increment for all growth parameters than the control except in root fresh weight was decreased when the dose 25 and $50 \mathrm{~g} / \mathrm{kg}$ in some treatment.

\section{(Animal and plant) compost tea 2 Days before inoculation and 10 Days after inoculation)}

Data in table (6) showed that, all treatments (animal and plant compost tea) were explicated with two time; two days before inoculation and ten days after inoculation. All treatments significantly affected Meloidogyne incognita development and could arrest its reproduction rate compared with check treatment. Concerning reduction percentage of juvenile's number, galls, females, egg-masses, eggs per egg masses, the final population and nematode build-up were diminished with all treatments. Animal compost tea treatment (2 days before inoculation) was better than the plant compost tea 2days before inoculation in all doses expect the dose of $100 \mathrm{~g} / 200 \mathrm{~cm} 3$ water plant compost tea which better than animal compost tea in final population. Also animal was compost tea 10 days after inoculation achieved best result than the plant compost tea 10 days after inoculation in final population.

On other hand the treatment with animal and plant compost tea 2 days before inoculation achieved the best result than that 10 days after inoculation. Also, the calculations of final population and rates of build -up were significantly affected by animal and plant compost tea 2 days before inoculation, the highest percentage of 
reduction in the nematode final population $(80.59 \%)$ and $(82.90 \%)$ was noticed in concentration dose100 $\mathrm{g} / 200 \mathrm{~cm}^{3}$ water $/ \mathrm{kg}$ while, the lowest reduction was $(69.94 \%)$ and $(65.69 \%)$ in concentration dose $25 \mathrm{~g} / 200 \mathrm{~cm}^{3}$ water $/ \mathrm{kg}$. All treatment at the highest concentration level achieved the lowest rate of build-up (1.19 folds) and (1.05 folds), resembling that obtained by the highest concentration dose 100 $\mathrm{g} / 200 \mathrm{~cm} 3$ water for $/ \mathrm{kg}$.

Accordingly, the calculated final population (fig., 2) and rates of build -up were significantly affected by animal and plant compost tea 10days after inoculation, the highest percentage of reduction in the nematode final population $(79.04 \%)$ and $(74.12 \%)$ was noticed in concentration dose $100 \mathrm{~g} / 200 \mathrm{~cm}^{3}$ water for each pot. while, the lowest reduction was (66.94\%) and $(57.97 \%)$ in concentration dose $25 \mathrm{~g} / 200 \mathrm{~cm}^{3}$ water $/ \mathrm{kg}$ all treatment at the highest concentration level achieved the lowest rate of build-up (1.28 folds) and (1.95folds), resembling that obtained by the highest concentration dose $100 \mathrm{~g} / 200 \mathrm{~cm} 3 w a t e r / \mathrm{kg}$.

Data on response of $M$. incognita -infected eggplant plants to drench application of (animal and plant) compost tea was tabulated in table (7). It was noticed that plant growth parameters, on basis of number of leaves, weights and length of both shoots and roots, highly responded to material applications, even with nematode infection. With regard to the influence of tested materials on shoot length no definite trend was noticed. Although, all materials used increased shoot lengths of treated plants, differences among values of such parameter were mostly insignificant. Moreover, plant and animal compost tea 10 days after inoculation and plant and animal compost tea 2 days before inoculation, was gave the best result $48.42,46.88 \%$ and $45.33 \%$ respectively compared to check. Also, these previous materials showed an increase in fresh weight that recorded $33, .78 \%, 29,91 \%$, and $47.89 \%$ respectively. Regarding to dry weight registered increase percentage were $80.57 \%, 71.01 \%$ and $63.37 \%$ respectively. While the dose $100 \mathrm{~g}$ in animal compost tea 2 days before inoculation gave the best result $82.88 \%$ in shoot dry weight, while the less result achieved by nematicide furan $20.70 \%$, also the dose of $25 \mathrm{~g} /$ $200 \mathrm{~cm}^{3}$ by plant compost tea10 days after inoculation and animal compost tea 2 days before inoculation recorded $41.08 \%$ and $45.54 \%$ respectively. Likely, root parameters had erratically respond to different concentration level of the tested materials. Most treatments which had significantly improved such parameters when compared to the check. However, with few exceptions as well as the dose 25 and $50 / 200 \mathrm{~cm} 3$ by plant compost tea10days after inoculation caused decreased in root fresh weight. 
Table (4): Effect of animal and plant compost different concentrations on Meloidogyne incognita development infected eggplant (Solanum melongena) under greenhouse condition.

\begin{tabular}{|c|c|c|c|c|c|c|c|c|c|c|c|c|c|c|c|c|}
\hline Treatments & $\begin{array}{l}\text { Dose / } \\
\text { pot (g) }\end{array}$ & $\begin{array}{c}\text { In } \\
\text { 60ll }\end{array}$ & $\% R$ & $\begin{array}{l}\text { Galls| } \\
\text { root }\end{array}$ & $\% R$ & $\begin{array}{c}\text { Eggg } \\
\text { mas5es/ } \\
\text { root }\end{array}$ & $\% R$ & $\begin{array}{l}\text { Eggsl } \\
909 \\
\text { mas6 }\end{array}$ & $\% R$ & Femalas & $\pi / \%$ & $\begin{array}{c}\text { dovelopmantal } \\
\text { stagos }\end{array}$ & $\% R$ & F.P & $\% R$ & R.B \\
\hline \multirow{3}{*}{$\begin{array}{l}\text { Animal } \\
\text { compost }\end{array}$} & 25 & $2340^{\circ}$ & 70.62 & $311^{4}$ & 81.83 & $211^{6}$ & 80.35 & $231^{6}$ & 3205 & $373^{6}$ & 85.47 & $322^{6}$ & 52.99 & 3246 & 73.57 & 1.62 \\
\hline & 50 & $1600^{\circ}$ & 7991 & $120^{\circ}$ & 9299 & $47^{4}$ & 95.62 & $207^{4}$ & 39.11 & $132^{4}$ & 94.85 & $47^{4}$ & 93.13 & 1826 & 85.14 & 91 \\
\hline & 100 & $883^{\circ}$ & 88.79 & $74^{4}$ & 95.67 & $39^{\prime}$ & 96.36 & $216^{* 4}$ & 36.47 & $74^{*}$ & 97.11 & $14^{\circ}$ & 97.95 & 1020 & 92.18 & 51 \\
\hline \multirow{3}{*}{$\begin{array}{c}\text { Plant } \\
\text { compost }\end{array}$} & 25 & $2383^{\circ}$ & 70.08 & $328^{\circ}$ & 80.89 & $290^{\circ}$ & 72.99 & $275^{\circ}$ & 19.11 & $425^{\circ}$ & 83.45 & $131^{6}$ & 80.87 & 3452 & 7192 & 1.72 \\
\hline & 50 & $1485^{\circ}$ & 81,36 & $105^{6}$ & 9386 & $62^{t}$ & 9422 & $183^{4}$ & 46.17 & $105^{54}$ & 9591 & $31^{\prime}$ & 95.47 & 1683 & 86.31 & 84 \\
\hline & 100 & $790^{\circ}$ & 90.08 & $62^{4}$ & 96.37 & $45^{2}$ & 95.81 & $138^{\circ}$ & 59.41 & $62^{\prime}$ & 97.58 & $19^{\circ}$ & 97.22 & 916 & 92.54 & .45 \\
\hline $\begin{array}{c}\text { Check1 } \\
\text { (Furan 10\%) }\end{array}$ & 2 & $2070^{\circ}$ & 74.01 & $372^{\circ}$ & 7827 & $176^{\circ}$ & 83.61 & $122^{\prime}$ & 64.11 & $37 \%$ & 85.51 & $112^{\circ}$ & 93.64 & 2554 & 79.10 & 1.27 \\
\hline Check2 & - & $7967^{2}$ & - & $1712^{4}$ & - & $1074^{2}$ & - & $340^{4}$ & - & $2568^{2}$ & - & $685^{2}$ & 80.90 & 12294 & - & 6.14 \\
\hline
\end{tabular}

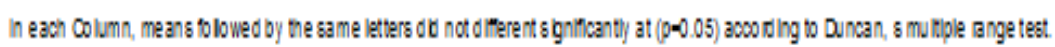

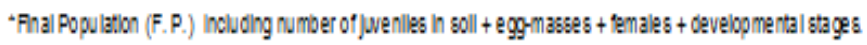


Evaluation of Compost and compost tea as promising method for Meloidogyne incognita management 58

Table (5): Plant growth parameters of eggplant (Solanum m elongena) affected by Meloidogyne incognita treated by different animal and plant compost concentrations under greenhous e conditions.

\begin{tabular}{|c|c|c|c|c|c|c|c|c|c|c|c|c|c|}
\hline \multirow[b]{2}{*}{ Treatnonts } & \multirow[b]{2}{*}{$\begin{array}{l}\text { Dosel } \\
\text { Pot (g) }\end{array}$} & \multicolumn{8}{|c|}{ Shoot } & \multicolumn{4}{|c|}{ Root } \\
\hline & & $\begin{array}{l}\text { Length } \\
\text { (cm) }\end{array}$ & $\%$ ncrea 59 & $\begin{array}{l}\text { Leave } \\
\text { number }\end{array}$ & \%/ncrease & $\begin{array}{c}\text { Fresh } \\
\text { Velght } \\
(g)\end{array}$ & \%lncrease & $\begin{array}{c}\text { Dry } \\
\text { Whight } \\
\text { (g) }\end{array}$ & \%lncrease & $\begin{array}{c}\text { Fresh } \\
\text { Velght } \\
\text { (g) }\end{array}$ & \%ancreas9 & $\begin{array}{l}\text { Longth } \\
\text { (cm) }\end{array}$ & \%/ncrease \\
\hline Animal & 25 & $28.66^{2}$ & 34.36 & $6.67^{\mathrm{c}}$ & 11.16 & $12.89^{4}$ & 7.56 & $4.50^{\mathrm{s}}$ & 43.31 & $4.30^{\circ}$ & 4.87 & $9.26^{4}$ & 0.75 \\
\hline \multirow[t]{3}{*}{ Compost } & 50 & $29.66^{2}$ & 39.05 & $9.00^{\circ}$ & 50 & $2027^{2 \mathrm{~b}}$ & 70.33 & $5.48^{x}$ & 7452 & $3.60^{d}$ & -12.19 & $11.00^{\circ}$ & 17.89 \\
\hline & 100 & $29.87^{4}$ & 40.03 & $9.00^{\circ}$ & 50 & $21.82^{2}$ & 83.36 & $592^{6}$ & 88.53 & 4.40 & 7.31 & $12.36^{2}$ & 32.47 \\
\hline & 25 & $24.66^{66}$ & 15.61 & $8.00^{*}$ & 33.33 & $14.07^{4}$ & 18.23 & $5.26^{x}$ & 67.51 & $3.34^{6}$ & -18.53 & $9.66^{6}$ & 3.53 \\
\hline \multirow[t]{2}{*}{$\begin{array}{c}\text { Plant } \\
\text { compost }\end{array}$} & 50 & $28.66^{4}$ & 34.36 & $7.33^{\text {tec }}$ & 22.16 & $15.99^{6 d}$ & 34.36 & $5.45^{x}$ & 73.56 & $3.62^{4}$ & -11.70 & $10.66^{\circ}$ & 14.25 \\
\hline & 100 & $30.33^{2}$ & 42.19 & $9.00^{\circ}$ & 50 & $18.26^{66}$ & 53.44 & $6.33^{2}$ & 101.59 & $4.73^{\circ}$ & 15.36 & $12.50^{2}$ & 33.97 \\
\hline $\begin{array}{c}\text { Check1 } \\
\text { (Furan 10\%) }\end{array}$ & 2 & $28.33^{2 b}$ & 32.81 & $8.00^{*}$ & 33.33 & $13.22^{4}$ & 11.09 & $3.76^{t}$ & 19.74 & $6.07^{4}$ & 48.04 & $9.00^{4}$ & -3.53 \\
\hline Check2 & - & $21.33^{6 *}$ & - & $600^{\circ}$ & - & $11.90^{4}$ & - & $3.14^{\prime}$ & - & $4.10^{\circ}$ & - & $9.33^{6}$ & - \\
\hline Healthy & - & $29.00^{2}$ & 35.95 & $7.67^{2 \mathrm{kC}}$ & 27.83 & $14.16^{\mathrm{s}}$ & 1899 & $5.11^{\omega \sigma}$ & 62.73 & $3.20^{\circ}$ & -7.31 & $10.83^{\circ}$ & 16.07 \\
\hline
\end{tabular}

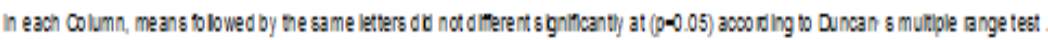


Table (8): Effect of different animal and plant compost tea treatments on Meloidogyne incognita development infected eggplant (Solanum melongena) under greenhouse condition.

\begin{tabular}{|c|c|c|c|c|c|c|c|c|c|c|c|c|c|c|c|c|}
\hline Treatnents & $\begin{array}{l}\text { Dosas } \\
\text { pot (g) }\end{array}$ & $\begin{array}{l}\text { Solll } \\
\text { pot }\end{array}$ & $\% R$ & $\begin{array}{l}\text { Gallsl } \\
\text { root }\end{array}$ & $\% R$ & $\begin{array}{c}\text { Egg } \\
\text { massol } \\
\text { root } \\
\end{array}$ & $\% R$ & $\begin{array}{l}\text { Eggsl } \\
\text { ggg } \\
\text { mas6 }\end{array}$ & $\% R$ & Fenalas & $\% R$ & $\begin{array}{l}\text { Dovelop } \\
\text { mantal } \\
\text { stagos } \\
\end{array}$ & $\% R$ & F.P & $\% R$ & RB \\
\hline \multirow{3}{*}{$\begin{array}{l}\text { A nilmal compost } \\
\text { ta } 2 \text { days before } \\
\text { Inocuation }\end{array}$} & 25 & $2970^{4}$ & 62.72 & $331^{\text {th }}$ & 80.66 & $228^{\circ}$ & 78.77 & $210^{d}$ & 38.23 & $397^{\circ}$ & 84.54 & $132^{\prime}$ & 80.72 & 3727 & 6994 & $1-86$ \\
\hline & 50 & $2340^{\circ}$ & 70.62 & $290^{\circ}$ & 83.06 & $180^{\circ}$ & 83.24 & $160^{\circ}$ & 52.94 & $319^{h}$ & 87.57 & $116^{\circ}$ & 83.05 & 2955 & 73.38 & 1.47 \\
\hline & 100 & $1960^{\circ}$ & 75.39 & $190^{k}$ & 8890 & $12 z^{k}$ & 88.64 & $9 g^{n}$ & 70.88 & 209 & 91.86 & $95^{n}$ & 86.13 & 2336 & 80.59 & 1. 19 \\
\hline \multirow{3}{*}{$\begin{array}{l}\text { Plant compost } \\
\text { ta } 2 \text { days bafore } \\
\text { Inocuation }\end{array}$} & 25 & $4185^{\circ}$ & 47.74 & $400^{\circ}$ & 76.63 & $288^{\circ}$ & 73.18 & $243^{6}$ & 28.53 & $520^{4}$ & 79.75 & $200^{6}$ & 70.88 & 5193 & 57.57 & 2.59 \\
\hline & 50 & $3360^{\circ}$ & 57.82 & $342^{\circ}$ & 80.02 & $264^{\circ}$ & 75.41 & $161^{\circ}$ & 52.64 & $410^{\circ}$ & 84.03 & $145^{\circ}$ & 78.39 & 4182 & 6598 & 2.09 \\
\hline & 100 & $1650^{\circ}$ & 79.28 & $234^{\prime}$ & 86.33 & $105^{k}$ & 90.13 & $129^{\circ}$ & 6205 & $255^{\prime}$ & 90.07 & $93^{n}$ & 86.42 & 2102 & 8290 & 1.05 \\
\hline \multirow{3}{*}{$\begin{array}{l}\text { Animal Compost } \\
\text { tea } 10 \text { dgy } 5 \text { a ter } \\
\text { Inocuation }\end{array}$} & 25 & $3327^{\circ}$ & 5824 & $360^{\circ}$ & 7897 & $270^{\circ}$ & 74.86 & $241^{\circ}$ & 29.11 & $491^{\circ}$ & 80.88 & $181^{d}$ & 73.57 & 4269 & 6527 & 2.13 \\
\hline & 50 & $2941^{\circ}$ & 63.08 & $308^{N}$ & 8200 & $209^{h}$ & 80.54 & $189^{\circ}$ & 4.41 & $400^{\circ}$ & 84.42 & $123^{\circ}$ & 82.04 & 3673 & 70.12 & 1.83 \\
\hline & 100 & $2167^{\prime \prime}$ & 7208 & $188^{k}$ & 89.07 & 148 & 86.21 & $129^{\circ}$ & 62.05 & 205 & 92.01 & $56^{\prime}$ & 91.82 & 2576 & 7904 & 1.28 \\
\hline \multirow{3}{*}{$\begin{array}{l}\text { plantcompost } \\
\text { taa } 10 \text { day } 6 \text { a ter } \\
\text { Inocuation }\end{array}$} & 25 & $3472^{4}$ & 56.42 & $654^{e}$ & 61.79 & $583^{2}$ & 45.71 & $305^{\circ}$ & 10.29 & $913^{8}$ & 64.44 & $262^{\circ}$ & 61.75 & 5230 & 57.45 & 2.61 \\
\hline & 50 & $2650^{\circ}$ & 66.73 & $564^{\circ}$ & 67.05 & $36 T^{\circ}$ & 65.82 & $198^{4}$ & 41.76 & $677^{4}$ & 73.63 & $169^{6}$ & 75.32 & 3868 & 6839 & 1.93 \\
\hline & 100 & $2050^{4}$ & 74.14 & $505^{d}$ & 70.50 & $313^{d}$ & 70.85 & $170^{\circ}$ & 50.00 & $556^{d}$ & 78.34 & $152^{\circ}$ & 77.81 & 3181 & 74.12 & 1.59 \\
\hline Check1(Furan) & 2 & $2070^{\prime}$ & 7401 & $372^{*}$ & 7827 & $176^{\prime}$ & 83.61 & $122^{\circ}$ & 64.11 & $372^{\circ}$ & 85.51 & $112^{\circ}$ & 83.64 & 2554 & 79.10 & 1.27 \\
\hline Check 2 & - & $7967^{4}$ & - & $1712^{2}$ & - & $1074^{2}$ & - & $340^{2}$ & - & $2568^{\circ}$ & - & $685^{4}$ & - & 12294 & - & 7.03 \\
\hline
\end{tabular}

In each Column, means followed by the same letters did not different significanty at $(p=0.05)$ according to Duncan, 5 multiple range test.

*Final Population (F. P.)Including number of juveniles in soil + egg-masses + females + developmental stages 
Table (7): Flant growth parameters of eggplant (Solanumm elongena) infected by Meloidogyne incognita and treated by compost tea (animal and plant) concentration under greenhouse conditions.

\begin{tabular}{|c|c|c|c|c|c|c|c|c|c|c|c|c|c|}
\hline \multirow[b]{2}{*}{ Treatments } & \multirow{2}{*}{$\begin{array}{l}\text { Dosal } \\
\text { pot }(g)\end{array}$} & \multicolumn{8}{|c|}{ shoot } & \multicolumn{4}{|c|}{ Root } \\
\hline & & $\begin{array}{l}\text { Longth } \\
\text { (cm) }\end{array}$ & $\begin{array}{c}\% \\
\text { Increas }\end{array}$ & $\begin{array}{c}\text { Lagvo } \\
\text { number }\end{array}$ & $\begin{array}{c}\% \\
\text { Increase }\end{array}$ & $\begin{array}{c}\text { Fresh } \\
\text { Velght } \\
\text { (g) }\end{array}$ & $\begin{array}{c}\% \\
\text { Increase }\end{array}$ & $\begin{array}{c}\text { Dry } \\
\text { Vhight } \\
\text { (g) }\end{array}$ & $\begin{array}{c}\% \\
\text { Increa } 69\end{array}$ & $\begin{array}{l}\text { Fresh } \\
\text { VWalght }\end{array}$ & $\begin{array}{c}\% \\
\text { Increas }\end{array}$ & $\begin{array}{l}\text { Longth } \\
\text { (cm) }\end{array}$ & $\begin{array}{c}\% \\
\text { Increase }\end{array}$ \\
\hline \multirow{2}{*}{$\begin{array}{l}\text { A nimal compost tea } \\
\text { 2days before }\end{array}$} & 25 & $25.33^{\mathrm{b}}$ & 18.75 & $7.67^{26}$ & 27.38 & $12.97^{\alpha b}$ & 8.99 & $4.57^{78 d}$ & 45.54 & $5.18^{\circ}$ & 26.34 & $9.66^{\mathbf{\Delta}}$ & 3.53 \\
\hline & 50 & $28.33^{*}$ & 24.70 & $7.67^{x d}$ & 27.38 & $15.65^{\text {bec }}$ & 31.51 & $5.42^{2 \mathrm{c}}$ & 7261 & $529^{4}$ & 29.02 & $11.50^{*}$ & 23.25 \\
\hline Inocuation & 100 & $30.00^{4}$ & 40.64 & $7.67^{28 d}$ & 27.38 & $14.56^{x}$ & 22.35 & $5.74^{2}$ & 8288 & $5.40^{\circ}$ & 31.70 & $12.00^{\circ}$ & 28.61 \\
\hline \multirow{3}{*}{$\begin{array}{l}\text { Plant composttea } \\
2 \text { day } 5 \text { before } \\
\text { Inoculation }\end{array}$} & 25 & $28.33^{*}$ & 24.70 & $7.33^{264}$ & 22.16 & $15.04^{2 \mathrm{ec}}$ & 26.38 & $4.75^{\text {ated }}$ & 51.27 & $3.84^{d}$ & -6.34 & $10.33^{\mathrm{tes}}$ & 10.71 \\
\hline & 50 & $28.33^{*}$ & 24.70 & $7.67^{28}$ & 27.38 & $15.97^{2 b}$ & 34.20 & $5.13^{2 *}$ & 63.37 & $393^{4}$ & -17.00 & $11.33^{*}$ & 21.43 \\
\hline & 100 & $31.00^{4}$ & 43.88 & $100.00^{2}$ & 66.66 & $17.60^{2}$ & 47.29 & $5.13^{* \mathrm{c}}$ & 63.37 & $4.30^{4}$ & 487 & $12.00^{4}$ & 28.61 \\
\hline \multirow{3}{*}{$\begin{array}{l}\text { Animal Compost toa } \\
10 \text { day } 5 \text { after } \\
\text { Inocuation }\end{array}$} & 25 & $29.00^{\circ}$ & 35.95 & $6.33^{24}$ & 5.50 & $15.40^{\text {etc }}$ & 29.41 & $5.68^{4}$ & 80.89 & $3.70^{d}$ & -9.75 & $10.66^{\text {stad }}$ & 14.25 \\
\hline & 50 & $30.66^{4}$ & 43.74 & $6.33^{24}$ & 5.50 & $16.52^{2 b}$ & 38.82 & $5.74^{2}$ & 8280 & $487^{\circ}$ & 18.78 & $11.16^{\mathrm{bec}}$ & 19.61 \\
\hline & 100 & $31.33^{4}$ & 46.88 & $7.33^{254}$ & 22.16 & $15.46^{\text {abc }}$ & 29.91 & $5.37^{* \mathrm{c}}$ & 71.01 & $5.09^{6}$ & 24.14 & $11.83^{*}$ & 26.79 \\
\hline \multirow{3}{*}{$\begin{array}{l}\text { plant compost tea } \\
10 \text { days after } \\
\text { Inocuation }\end{array}$} & 25 & $26.00^{\circ}$ & 21.29 & 6.00 & - & $14.55^{x}$ & 22.25 & $4.43^{66}$ & 41.08 & $6.41^{\circ}$ & 56.34 & $10.83^{\text {teds }}$ & 25.79 \\
\hline & 50 & $31.33^{4}$ & 43.74 & $6.67^{2.4}$ & 11.16 & $15.31^{2 \mathrm{Ec}}$ & 28.65 & $5.50^{28}$ & 75.15 & $6.76^{*}$ & 64.87 & $10.66^{\mathrm{sed}}$ & 14.25 \\
\hline & 100 & $31.66^{4}$ & 48.42 & $8.00^{66}$ & 33.33 & $15.92^{2 \mathrm{~b}}$ & 33.78 & $567^{4}$ & 80.57 & $7.25^{4}$ & 76.82 & $12.17^{4}$ & 13.43 \\
\hline Check1(Furan) & 2 & $28.33^{*}$ & 32.81 & $8.33^{b}$ & 38.83 & $13.22^{4}$ & 11.09 & $3.79^{4}$ & 20.70 & $6.07^{\circ}$ & 480.4 & $9.00^{4}$ & 3.5 \\
\hline Check2 & - & $21.33^{6 *}$ & - & $6.00^{4}$ & - & $1190^{\circ}$ & - & $3.14^{*}$ & - & $4.10^{\circ}$ & - & $9.33^{\mathrm{A}}$ & - \\
\hline Hea ithy & - & $29.00^{*}$ & 35.95 & $7.67^{x d}$ & 27.38 & $14.17^{\text {tod }}$ & 19.07 & $5.11^{* c}$ & 62.73 & $3.80^{d}$ & -7.31 & $10.83^{\text {ted }}$ & 19.61 \\
\hline
\end{tabular}

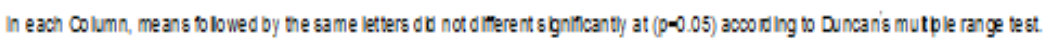




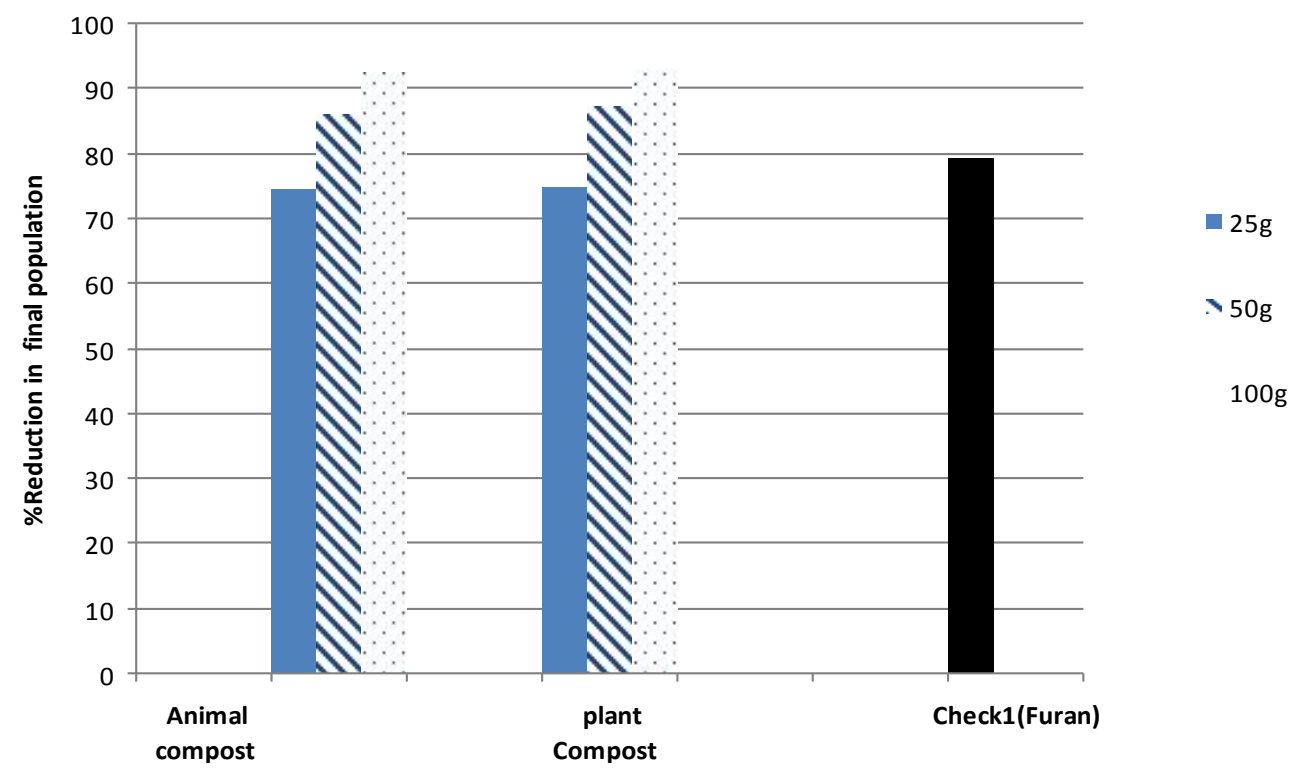

Fig. (1): \%Reduction in final population of Meloidogyne incognita infected eggplant roots and influenced by two type of Compost.

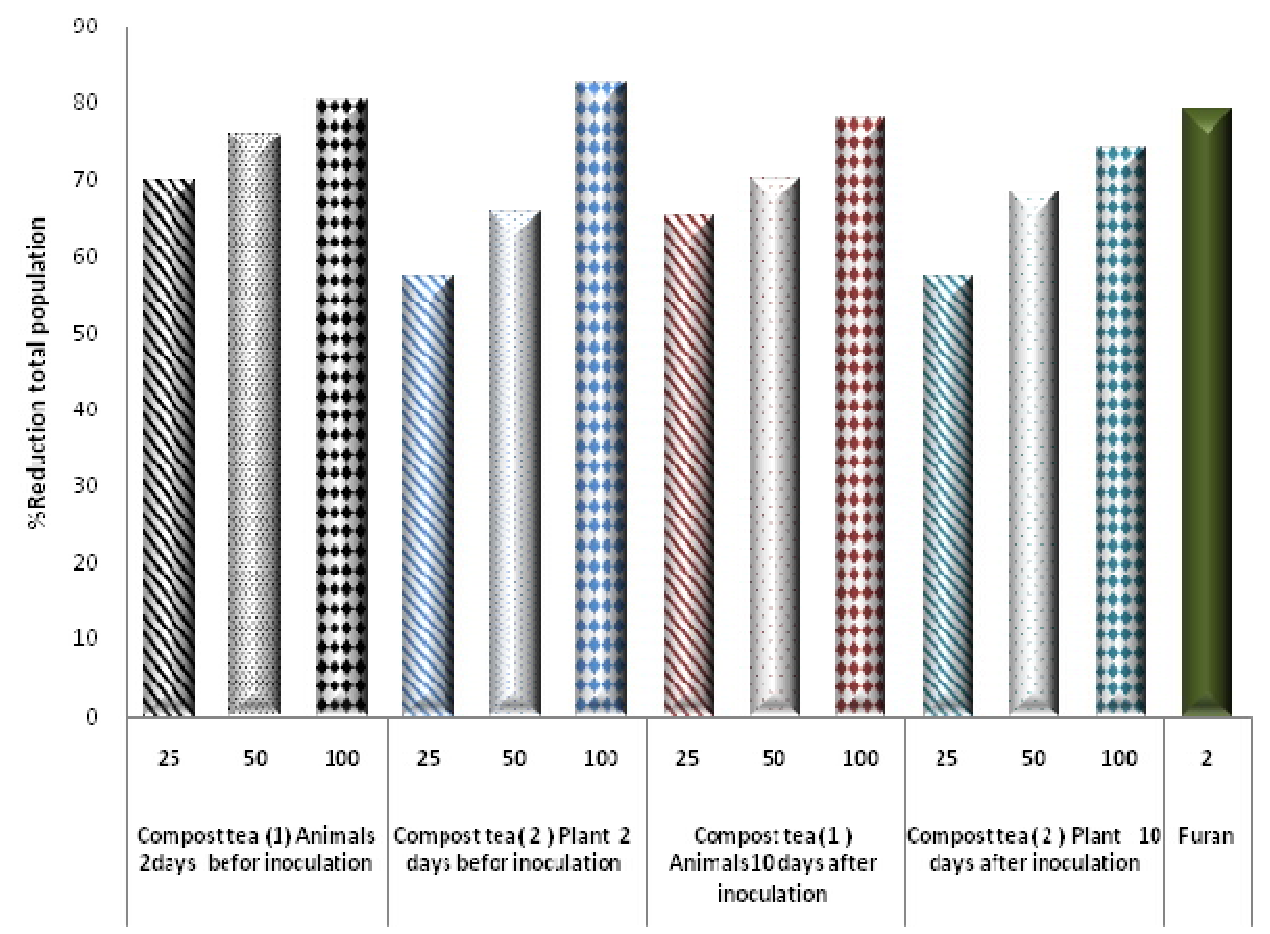

Fig. (2): \%Reduction in total population of Meloidogyne incognita infected eggplant roots influenced by Compost tea. 


\section{Discussion}

The efficacy of compost seemed to be depends on the components of which it were produced, so differences were observed in the present work among the two tested types of compost (plant and animal) the present study are harmony with those of Moselhy (2009) who reported that compost significantly reduced formation of galls on sunflower roots, the developmental stages and the final population nematode many reports indicated that compost application improved growth of infected plants and diminished nematode population (Leroy at el.,2007 and Cayuela et al.,2008). The beneficial effects of compost on treated plants rather than its role in controlling nematode were outlined by many authors they mentioned that compost improves soil structure, porosity; increases infiltration and permeability of heavy soils, increases water holding capacity, supplies variety of macro and micronutrients; supplies significant quantities of organic matter, improves cation exchange capacity. Also, compost stabilizes soil $\mathrm{PH}$, provides humus, vitamins, hormones, and plant enzymes which are not supplied by chemical fertilizers (Field guide to compost use, 2001; Evanylo et al., 2008; Ahamed et al., 2009).

The mechanisms by which composts suppress plant diseases and plant parasitic nematodes are still speculative but it may be due to increase competition from fungivorous and bacterivorous nematodes resulting from increased availability of food sources after compost applications. There is good evidence (Edwards, 1998) that earthworms greatly increase overall microbial activity in organic wastes greatly by providing fragmented organic materials for microbial growth of soil bacteria and fungi. Soils that were treated with inorganic fertilizers only had much less organic matter available for microbial growth compared to those in the Compost treated soils. The effects of compost applications on soil were much greater on fungivorous nematode populations than on bacterivorous nematode populations. As it is know, Earthworms depend upon fungi as a main source of food and tend to increase fungal activity in their casts by excreting fungal spores (Edwards \& Fletcher, 1988) which may also explain why there were greater increases in populations of fungivorous nematodes than in those of bacterivorous nematodes. Moreover, plant parasitic nematodes are attacked by cyst fungi and nematode trapping fungi populations of which could have increased in response to compost applications (Kerry, 1988). The greater availability of microorganisms as a source of energy could increase the competitive ability of both bacterivorous and fungivorous nematodes as compared to plant parasitic nematodes.

\section{References}

Addabbo, D.; Papajova,T.; Sasanelli, I.; Radicci.; N and Renco, V.M. (2011). Suppression of root-knot nematodes in potting mixes amended with different composted biowastes. Helminthologia., 48(4): 278-287 
Ahmad, F. and Siddiqui, M.A. (2009). Management of root-knot nematode, Meloidogyne incognita in tomato. Pak. J. Nematol., 27: 369-373.

Ahmed, N.; Abbasi, M.W.;Shaukat, S.S. and Zaki M.J. (2009). Induced Systemic Resistanc in Mung Bean plant against Root-Knot Nematode Meloidogyne Javanica by DI-B-Amino Butyric Acid Nematol. medit., 37: 67-72 67.

Cayuela, M.I.; Millner, P.D.; Myer, S.L. and Rog, A. (2008). Potential of olive mill waste and compost as biobased pesticides against weeds, fungi and nematodes. Sic. Total Environ. 309;11-18

Edwards, C A. (1998). Breakdown of animal, vegetable and industrial organic wastes by earthworms. In: Earthworm Ecology, pp 237-354. CRC.

Edwards, C. A. and Fletcher K E. (1988). Interactions between earthworms and microorganisms in organic matter breakdown. Agriculture, Ecosystems and Environment, 20 (3):235-249.

Evanylo, C.; Sherony, C.; Spargo, J.; Starner, D.; Brosius, M. and Haering, K. (2008). Soil and water environmental effects of fertilizer, manure and compost-based fertility practices in an organic vegetable cropping system. Agric. Ecosys. Environ., 127:50-58.

Farahat, A. A.; Al-Sayed A.A. and Mahfoud, N.A. (2010). Compost and other Organic and Inorganic Fertilizizers in the Scope of the root-knot Nematode Reproduction and Control. Egypt, J. Agronematol.,9 (1):18-29.

Field Guide to compost Use. (2001). Guide to compost Use. U.S. Composting Council, 1ComacLoop, Suite 14B1 Ronkonkoma, NY, 11779.

Ibrahim, I.K.A.; Handoo, Z.A. and El-Sherbiny, A.A. (2000). A survey of Phytoparasitic nematodes on cultivated and non-cultivated plants in Northwestern Egypt.J.Nematol.,32: 478-485.

Kerry, B.R. (1988). Fungal parasites of cyst nematodes. Agric. Ecosyst. Environ. 24:293-305.

Leroy, M.; Bommele, L.; Reheul, D.; Mones, M. and De Neve, S. (2007).The application of vegetable, fruit and garden waste (VFG)c compost in addition to cattle slurry in asilage maze monculture effects on soil fauna and yiled European J. of Soil Biology, 43:91-100.

McSorley, R. and Gallaher, R. (1995).Effect of Yard waste compost on nematode densities and maize yield. J. Nematol., 28 (45): 655-660.

McSorley, R. and Gallaher, R. (1996). Effect of Compost and maize cultivars on plant parasitic nematode J. Nematol., 29 (45): 731-736. 
McSorley, R. and Gallaher, R. (1997).Effect of Yard waste compost on plant parasitic nematode densities in vegetable crops. J. Nematol., 27 (45): 545549.

Meyer, S. L. F.; Orisajo, S. B.; Chitwood, D. J.; Vinyard, B. T. and Millner, P. D.(2011). Poultry litter compost for suppression of root-knot nematode on cacao plants. International J. of Nematology, 21(2): 153-162.

Moselhy, M.A. (2009). Bioconversion of rice straw and certain agro-industrial wastes to fertilizers for organic farming systems. Ph.D. Thesis. Fac., of Agric., Cairo Univ. pp 221.

Nico, A.I.; Jimenez-Diaza, R.M. and Castillo, P. (2004). Control of root -knot nematodes by composted aggroindustrial wastes in potting mixtures. Crop Protection, 23:581-587.

Oka, Y., Koltai, H.; Bar-Eyal, M.; Mor, M,; Sharon E., Chet, I. and Spiegel, Y. (2000). New strategies for the control of plant-parasitic nematodes. Pest Management Science, 56: 983-988.

Ravindra, H.; Sehgal, M.; Pawan, A.S.; Archana, B.S.; Shruti, S.A. and Narasimhamurty, H. B. (2014). Eco-friendly management of root-knot nematodes using acacia compost and bioagents in brinjalPakistan Journal of Nematology, 32:1: 33-38.

Renco, M.; Sasanelli, N.; D'Addabbo, T. and Papajova, I. (2010). Soil nematode community changes associated with compost amendments. Nematology, 12: 5, 681-692

Rich, J.R., R. Dunn and J. Noling, (2004). Nematicides: Past and present uses. Nematology: Advances and Perspectives, 2: 1041 -1082.

Sasanelli, N.; D'Addabbo, T. and Mancini, L. (2011). Suppressive effect of composted olive mill wastes soil amendments on the root-knot nematode Meloidogyne incognita. Acta Horticulturae, 914:229-231.

Schneider, S.M.; Rosskopf, E.N.; Leesch J.G.; Chellemi, D.O.; Bull, C.T. and Mazzola, M. (2003). Research on alternatives to methyl bromide: pre-plant and post-harvest. PestManag Sci., 59(6-7): 814-826.

Sendecor, G.W. and Cochran, W.G.(1980). Statistical methods. Oxford \& J.BH Publishing com. $7^{\text {th }}$.edition.

Southey, J.F., ed. (1970). Laboratory Methods for Work with Plant and Soil Nematodes.MinistryAgricultureal Fish. And Food Tech. Bulletin 2. Her Majesty's Stationery Office Iondon.148Pp. 
Zhang -Shuang, Xi and Zhang xing (2009). Effects of two composted plant pesticide residues incorporated with Trichoderma virideon root -knot nematode in ballon flower. Agricultural Sciences in China,8(4):447-454.

Zhu, K.J.; Wang, B.; Fang, W.Z. and luo, D.M. (2006). Suppressive capacity of compost extracts and compost tea against the root-knot nematode Meloidogyne javanica on tomato in potting testes. J. of Yangtze, 1:116-118. 


\section{الملخص العربي}

تقييم فعالية الكمبوست وشاي الكمبوست كوسيلة واعدة لمكافحة نيماتودا تعقد الجذور

$$
\begin{aligned}
& \text { نجوى عبد الحميد عبد الباري*، حسن حامد هندي**، عزة هاشم عشوب**، }
\end{aligned}
$$

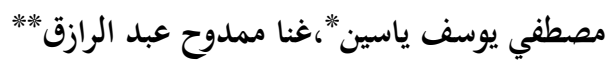

$$
\begin{aligned}
& \text { "قسم الحيوان والنيماتولوجيا الزراعية، كلية الزراعة، جامعة القاهرة، الجيزة }
\end{aligned}
$$

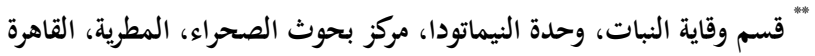

تم اختبار فعالية نوعيين من الكمبوست (حيواني ونباتي) بالإضافة إلى المبيد فيوران

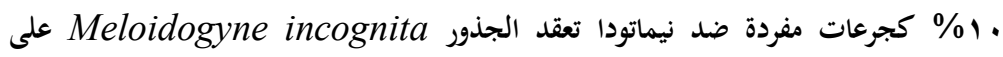

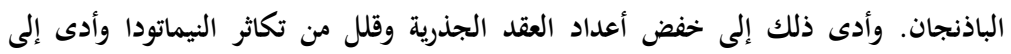
تحسين نمو النبات.

وتم إجراء هذه الدراسة تحت ظروف المعمل والبيوت الزجاجية عن طريق استخدام الكمبوست المنتج في مزارع سيكم العضوية. وتم تحضير مستخلص الكمبوست عن طريق إذابة الكرول

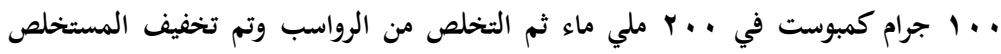

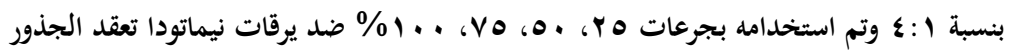
Meloidogyne incognita

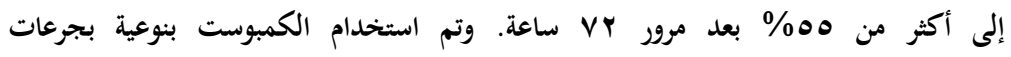

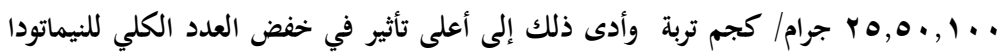

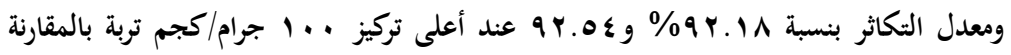

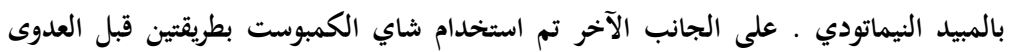

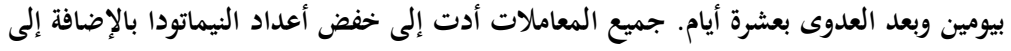

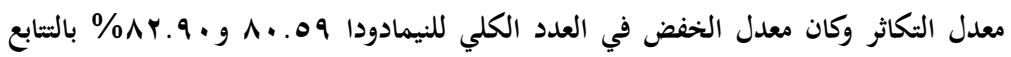

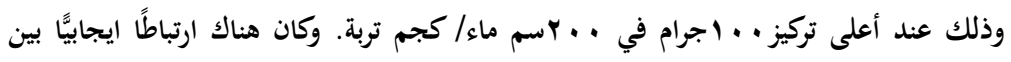

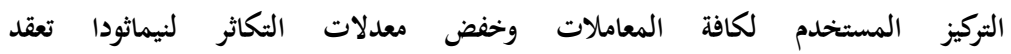

الجذور Meloidogyne incognita. 